\title{
Assembly of Poly(vinyl alcohol) and DNA via Hydrogen Bonds Induced by High Hydrostatic Pressurization
}

\author{
Tsuyoshi Kimura*, Akira Okuno ${ }^{1}$, Yuichi Ohya ${ }^{1}$ and Akio Kishida \\ Institute of Biomaterials and Bioengineering, Tokyo Medical and Dental University, \\ 2-3-10 Kanda-surugadai, Chiyoda-ku, Tokyo 135-0021, Japan \\ ${ }^{1}$ Department of Chemistry and Materials Engineering, Kansai University, \\ 3-3-35 Yamate-cho, Suita, Osaka 564-8680, Japan
}

(Received January 29, 2014; accepted July 16, 2014)

Key words: high hydrostatic pressure, DNA, poly(vinyl alcohol), hydrogen bond, assembly

According to our previous research, it was found that the assemblies of poly(vinyl alcohol) (PVA) and DNA, such as nanoparticles, microparticles and hydrogels, were formed through a high hydrostatic pressure process (10000 atm, $10 \mathrm{~min}$ ) and could be applicable for gene delivery. In the present study, the PVA-DNA assembly was characterized by various methods, such as agarose gel electrophoresis, melting point and circular dichroism measurements, atomic force microscopy (AFM) observation and nuclease digestion assay. From agarose gel electrophoresis and melting point measurement of the PVA-DNA assembly, it was clarified that the interaction between them was hydrogen bonding. The B-type form of DNA of the PVA-DNA assembly was shown by circular dichroism measurement. The increase in width of DNA in the PVADNA assembly was observed by AFM. The increase in nuclease resistance of the PVADNA assembly was shown. From these results, it was suggested that the PVA was entwined with the DNA duplex via hydrogen bonds under high hydrostatic pressure.

\section{Introduction}

Molecular assembly technology has been gathering interest in the material processing fields. Molecular assembly is achieved by noncovalent bonding between adjacent molecules. The development of carbon nanotubes as circuit wires ${ }^{(1,2)}$ and the incorporation of anticancer drugs and amphiphilic polymers into nanomicelles ${ }^{(3,4)}$ are examples of molecular assembly in which noncovalent bonding, such as electrostatic interaction, van der Waals interactions and hydrogen bonds, are well combined. ${ }^{(5-8)}$ Controlling these intermolecular forces is the key factor for creating or collapsing the assembled structure. Supramolecular chemistry has expanded to allow various elemental molecules to generate elegant assemblies, ${ }^{(9-12)}$ whereas the operative factors, which

*Corresponding author: e-mail: kimurat.mbme@tmd.ac.jp 
regulate molecular assembly, are mostly limited by the concentration and/or temperature. Previously, we focused on pressure, which is one of the most intensive variables in thermodynamics, as well as the concentration and temperature, ${ }^{(13-16)}$ and reported that the assembly of poly(vinyl alcohol) (PVA) via hydrogen bonds was induced under high hydrostatic pressure, ${ }^{(17)}$ resulting in the formation of nanoparticles, microparticles and hydrogels. Also, we have reported that the molecular assembly of PVA and DNA could be formed by high hydrostatic pressurization (HHP) and they could be applicable for gene delivery. ${ }^{(18-20)}$ In the present study, to clarify how PVA-DNA assembly induced by HHP is formed, the PVA-DNA assembly was investigated in detail using various methods, such as agarose gel electrophoresis, melting point and circular dichroism measurements, atomic force microscopy (AFM) observation and nuclease resistance assay.

\section{Materials and Methods}

\subsection{Materials}

We used PVA with a degree of polymerization of 1700 and a degree of saponification of $99.8 \%$. It was kindly supplied by Kuraray Co., Ltd. (Okayama, Japan). DNA molecular weight marker (1 kbp ladder, 1-10 kbp) was purchased from Takara Bio Inc. (Otsu, Japan). DNase I was purchased from Roche Diagnostics Japan K. K. (Tokyo, Japan). Ethidium bromide (EtBr) was obtained from Nippon Gene (Tokyo, Japan).

\subsection{Preparation of PVA-DNA assembly by HHP treatment}

PVA solutions of $2 \mathrm{w} / \mathrm{v} \%$ were prepared by autoclaving at $121{ }^{\circ} \mathrm{C}$ for $30 \mathrm{~min}$ three times and diluted to various concentrations $(0.0002-0.2 \mathrm{w} / \mathrm{v} \%)$. DNA solutions were prepared in TE buffer (10 mM Tris-HCl, $1 \mathrm{mM}$ EDTA, $\mathrm{pH}$ 8.0) at a concentration of 0.002 $\mathrm{w} / \mathrm{v} \%$. They were mixed at equal volumes and hydrostatically pressurized at 2000 and 10000 atmospheres and at $40{ }^{\circ} \mathrm{C}$ for 10 min using a high hydrostatic pressure machine (Dr. Chef, Kobe steel, Co. Ltd., Kobe, Japan).

\subsection{Agarose gel electrophoresis of PVA-DNA assembly prepared by HHP treatment}

The assembly of DNA and PVA produced by HHP was examined by agarose gel electrophoresis. The HHP-treated samples were electrophoresed through $1.0 \mathrm{w} / \mathrm{v} \%$ agarose gels at $100 \mathrm{~V}$ and $4{ }^{\circ} \mathrm{C}$ for $1 \mathrm{~h}$. The gel was stained with $\mathrm{EtBr}$ and observed under a UV transilluminator.

For the experiments described below, the assembly of DNA (final conc., $0.001 \mathrm{w} / \mathrm{v} \%$ ) and PVA (final conc., $0.001 \mathrm{w} / \mathrm{v} \%$ ) obtained by HHP treatment at $10000 \mathrm{~atm}$ was used.

\subsection{Melting temperature measurement of PVA-DNA assembly prepared by HHP treatment}

The melting profile of the DNA-PVA assembly was investigated by absorbance at $260 \mathrm{~nm}$ using a UV-vis spectrometer (V-550, Jasco Co., Tokyo, Japan) equipped with a Peltier EHC363-type cell holder (Jasco Co., Tokyo, Japan). The sample was heated from 
35 to $80{ }^{\circ} \mathrm{C}$ at a heating rate of $2.0{ }^{\circ} \mathrm{C} / \mathrm{min}$. These experiments were carried out three times.

\subsection{Circular dichroism measurement of PVA-DNA assembly prepared by HHP treatment}

The secondary structure of DNA assembled with PVA was also investigated using a circular dichroism (CD) spectrometer (J-720W, Jasco Co., Tokyo, Japan). The DNAPVA assembly was characterized five times to obtain the average spectra. These experiments were carried out three times.

\subsection{AFM observation of PVA-DNA assembly prepared by HHP treatment}

The morphology of the PVA-DNA assembly was observed by AFM. Ten microliters of the solution of the PVA-DNA assembly was dropped onto a mica surface and left for about $5 \mathrm{~min}$. The specimen was thoroughly rinsed twice with deionized water, and then dried with compressed air. Typical AFM images were acquired in tapping mode with silicon cantilevers (spring constant, 0.6-6.0 N/m) using the SPA-300 scanning probe microscope (Seiko Instruments, Tokyo, Japan). All the AFM images were raw data except flattening.

\subsection{Nuclease resistance of PVA-DNA assembly prepared by HHP treatment}

To investigate DNA degradation by DNase I, after the addition of $0.06 \mathrm{ml}$ of DNase I $(0.1 \mathrm{mg} / \mathrm{ml})$ to $0.54 \mathrm{ml}$ of the solution of the DNA-PVA assembly containing $\mathrm{MgCl}_{2}$ (final concentration of $5 \mathrm{mM}$ ) at $37{ }^{\circ} \mathrm{C}$, the absorbance at $260 \mathrm{~nm}$ was measured using a UVvis spectrometer. These experiments were carried out three times.

\section{Results}

\subsection{Agarose gel electrophoresis of PVA-DNA assembly}

PVA solutions $(0.0002-0.2 \mathrm{w} / \mathrm{v} \%)$ were mixed with a DNA solution (1 kbp ladder, $0.002 \mathrm{w} / \mathrm{v} \%$ ) at equal volumes and hydrostatically pressurized at 2000 or $10000 \mathrm{~atm}$ and at $40{ }^{\circ} \mathrm{C}$ for $10 \mathrm{~min}$. After pressure was removed, they were analyzed by agarose gel electrophoresis to investigate the assembly of DNA with PVA (Fig. 1). DNA bands in the mixed solutions of PVA and DNA without the hydrostatic pressurization at 2000 and $10,000 \mathrm{~atm}$ were observed as well as that in the DNA solution without PVA, regardless of the PVA concentration [Figs. 1(a) and 1(b)]. For all samples pressurized at 2,000 atm, there was no difference in electrophoretic patterns of the nonpressurized DNA [Fig. 1(a)]. In the case of the HHP treatment of 10,000 atm, smearing of DNA to the entire lane was observed at each PVA concentration [Fig. 1(b)], indicating the assembly of PVA and DNA. In particular, DNA bands over $5 \mathrm{kbp}$ showed smearing, but DNA bands lower than $5 \mathrm{kbp}$ were clearly visible. To investigate whether the assembly of PVA and DVA was mediated by hydrogen bonding interaction, the mixed solutions of DNA and PVA with urea $(1.0 \mathrm{M})$, which was used as a hydrogen bond inhibitor, were hydrostatically pressurized at $10000 \mathrm{~atm}$ and analyzed by agarose gel electrophoresis [Fig. 1(c)]. No DNA smearing was observed, regardless of PVA concentration and HHP treatment at $10000 \mathrm{~atm}$. 

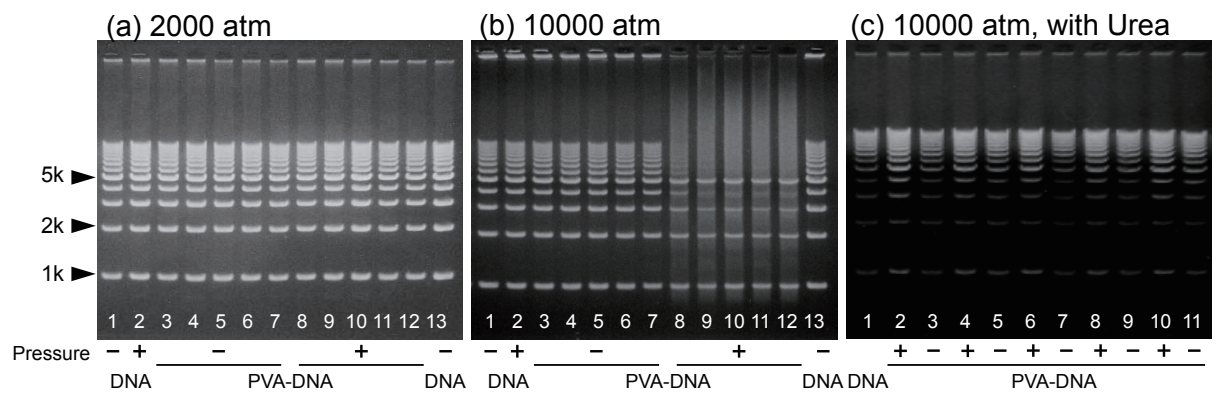

Fig. 1. Agarose gel electrophoresis of mixed solutions of PVA and DNA treated with high hydrostatic pressure at (a) $2000 \mathrm{~atm}$ and (b), (c) $10000 \mathrm{~atm}$ and at $40{ }^{\circ} \mathrm{C}$ for $10 \mathrm{~min}$. In panels (a) and (b), lanes 1, 13: nontreated DNA without PVA, lane 2: HHP-treated DNA without PVA, lanes 3-7: noHHP-treated DNA with PVA, lanes 8-12: HHP-treated DNA with PVA. PVA conc.: lanes 3, 8: 1.0\%, lanes 4, 9: 0.1\%, lanes 5, 10: 0.01\%, lanes 6, 11: 0.001\%, lanes 7, 12: 0.0001\%. In panel (c), lane 1: nontreated DNA without PVA, lanes 2, 4, 6, 8, 10: HHP-treated DNA with PVA, lanes 3, 5, 7, 9: non-HHP-treated DNA with PVA. PVA conc.: lanes 2, 3: 1.0\%, lanes 4, 5: 0.1\%, lanes 6, 7: 0.01\%, lanes 8, 9: 0.001\%, lanes 10, 11: 0.0001\%. DNA conc.: $0.001 \mathrm{w} / \mathrm{v} \%$.

\subsection{Tm measurement of PVA-DNA assembly}

The PVA-DNA assembly prepared by the HHP treatment at 10000 atm was investigated through melting curve measurement of DNA. Melting of double-stranded DNA can be investigated on the basis of an increase in absorbance (hyperchromic effect) that results from the disruption of base stacking in the DNA duplex owing to the breakage of hydrogen bonds. Figure 2 shows melting profiles of DNA solutions with and without the HHP treatment at $10000 \mathrm{~atm}$ in the absence or presence of PVA. The melting profiles of the nontreated DNA in the absence or presence of PVA were monophasic and their melting temperatures (Tm) were obtained at approximately $57 \pm 3{ }^{\circ} \mathrm{C}$ and 58 $\pm 2{ }^{\circ} \mathrm{C}$, respectively. For the HHP-treated DNA without PVA, the relative absorbance gradually decreased with increasing temperature between 50 and $60{ }^{\circ} \mathrm{C}$, and $\mathrm{Tm}$ of $62 \pm$ $2{ }^{\circ} \mathrm{C}$ was shown. In the case of the PVA-DNA assembly prepared by the HHP treatment at $10000 \mathrm{~atm}$, the melting profile was biphasic, that is, the relative absorbance gradually increased between 40 and $50{ }^{\circ} \mathrm{C}$ and subsequently increased suddenly at about $53 \pm 2{ }^{\circ} \mathrm{C}$.

\subsection{CD analysis of PVA-DNA assembly}

The secondary structure of DNA was examined by CD. Figure 3 shows the typical CD spectra of DNA and the PVA-DNA mixture with and without the HHP treatment at $10000 \mathrm{~atm}$. The CD spectra of the nontreated DNA displayed a positive peak at approximately $280 \mathrm{~nm}$ and a negative peak at approximately $246 \mathrm{~nm}$, indicating B-DNA conformation. In the case of the HHP-treated DNA without PVA, the negative and positive peaks were shifted to approximately 240 and $290 \mathrm{~nm}$, respectively. The cotton effect was enhanced. In the presence of PVA, a similar CD spectrum was obtained, regardless of the HHP treatment. The negative and positive peaks were shifted to approximately 245 and $295 \mathrm{~nm}$, respectively. The cotton effect was enhanced. 

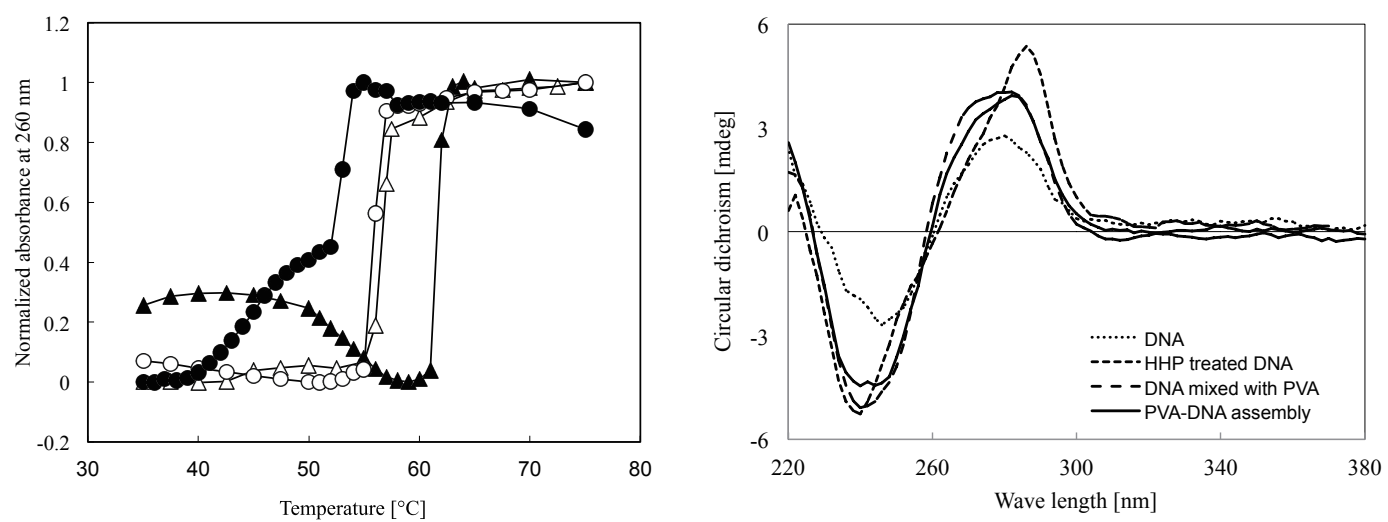

Fig. 2 (left). Melting curves of nontreated DNA (open triangle), HHP-treated DNA (closed triangle), DNA mixed with PVA without HHP treatment (open circle) and PVA-DNA assembly induced by HHP treatment (closed circle). HHP treatment conditions: $10000 \mathrm{~atm}, 40{ }^{\circ} \mathrm{C}, 10 \mathrm{~min}$. PVA conc., $0.001 \mathrm{w} / \mathrm{v} \%$; DNA conc., $0.001 \mathrm{w} / \mathrm{v} \%$.

Fig. 3 (right). CD spectrum of nontreated DNA (dot line), HHP-treated DNA (short dashed line), DNA mixed with PVA without HHP treatment (long dashed line) and PVA-DNA assembly induced by HHP treatment (solid line). HHP treatment conditions: $10000 \mathrm{~atm}, 40{ }^{\circ} \mathrm{C}, 10 \mathrm{~min}$. PVA conc., 0.001 w/v\%; DNA conc., $0.001 \mathrm{w} / \mathrm{v} \%$.

\subsection{AFM observation of PVA-DNA assembly}

Figure 4 shows AFM images of the nonpressurized DNA and PVA-DNA assembly prepared by HHP treatment. The nonpressurized DNA was displayed as a straight line [Fig. 4(a)]. The width of the DNA chain was about $32.2 \mathrm{~nm}$. For the PVA-DNA assembly (PVA concentration: $0.001 \%$ ), the bended DNA chain and the particle were observed [Fig. 4(b)]. The width of the DNA chain in the absence of the particle was about $37 \mathrm{~nm}$. In another observation field of the DNA-PVA assembly, many particles were observed [Fig. 4(c)]. These particles are PVA-DNA assemblies and assemblies of PVA molecules, which were reported previously. ${ }^{(18)}$ The globule on the straight line of DNA was also observed. The width of the PVA-DNA assembly slightly increased compared with that of the nontreated DNA.

\subsection{Nuclease resistance of PVA-DNA assembly}

Nuclease digestion test of the PVA-DNA assembly was carried out using DNase I (0.01 $\mathrm{mg} / \mathrm{ml}$ ). Figure 5 shows the time course of the absorbance of samples at $260 \mathrm{~nm}$.

After the addition of DNase I to the nontreated DNA solution and DNA solution containing PVA, the absorbance of the solution increased immediately owing to the fragmentation of the DNA. For the HHP-treated DNA without PVA, the absorbance at $260 \mathrm{~nm}$ was immediately increased and became constant, and the degree of the delta absorbance was lower than that of the nontreated DNA with and without PVA. In the case of the PVA-DNA assembly, the absorbance at $260 \mathrm{~nm}$ gradually increased and became constant. A small difference in the absorbance change was shown among them. 


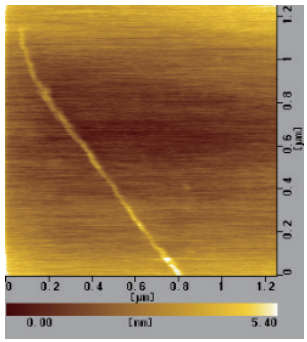

(a)

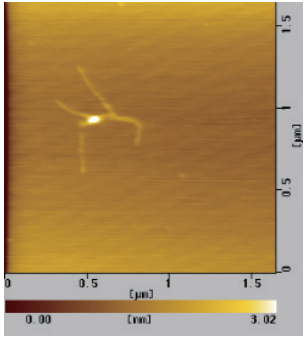

(b)

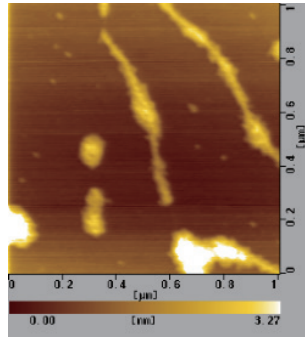

(c)

Fig. 4. (Color online) AFM images of (a) nontreated DNA, (b) and (c) PVA-DNA assembly induced by HHP treatment. HHP treatment conditions: $10000 \mathrm{~atm}, 40{ }^{\circ} \mathrm{C}, 10 \mathrm{~min}$. PVA conc., 0.001 w/v\%; DNA conc., $0.001 \mathrm{w} / \mathrm{v} \%$.

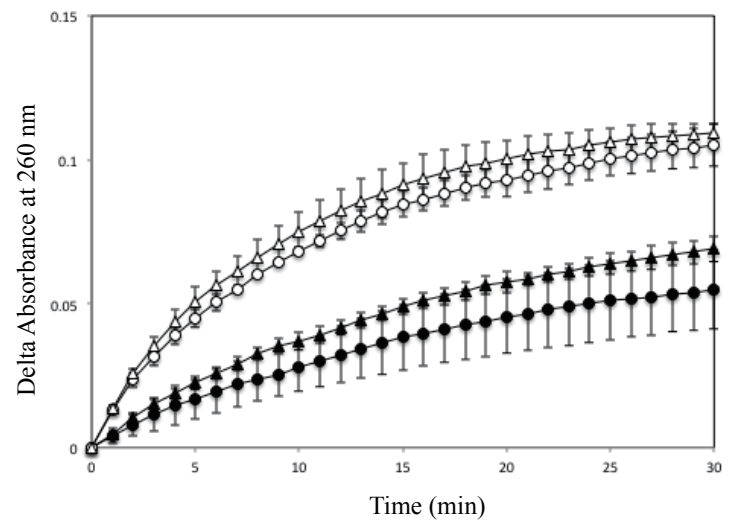

Fig. 5. Nuclease digestion assays for nontreated DNA (open triangle), HHP-treated DNA (closed triangle), DNA mixed with PVA without HHP treatment (open circle) and PVA-DNA assembly induced by HHP treatment (closed circle). HHP treatment conditions: $10000 \mathrm{~atm}, 40{ }^{\circ} \mathrm{C}, 10 \mathrm{~min}$. PVA conc., $0.004 \mathrm{w} / \mathrm{v} \%$; DNA conc., $0.004 \mathrm{w} / \mathrm{v} \%$.

\section{Discussion}

The PVA-DNA assembly prepared by the HHP treatment was characterized by various methods. Firstly, the interaction of PVA and DNA was investigated. By agarose gel electrophoresis of the mixed solution of PVA and DNA pressurized at 10,000 atm, the formation of the PVA-DNA assembly was confirmed. In the presence of urea, the assembly of PVA and DNA was inhibited. These results indicate that the interaction between PVA and DNA was mediated via hydrogen bonds. For Tm measurement, the melting points of DNA with and without the HHP treatment were $62 \pm 2$ and $57 \pm 3{ }^{\circ} \mathrm{C}$, respectively, indicating the stabilization of DNA by the HHP treatment. It was reported that the twisting and winding of the DNA helix was increased under a high-hydrostaticpressure condition, resulting in the high stability of the DNA helix. ${ }^{(21,22)}$ In the case of the PVA-DNA assembly, the gradual increase in the relative absorbance between 40 and 50 
${ }^{\circ} \mathrm{C}$ was shown, and Tm of the DNA helix was $53 \pm 2$. It is considered that the hydrogen bonding interaction of PVA and DNA was gradually dissociated with increasing temperature, and subsequently, the DNA double strand was dissociated. This revealed that the interaction between PVA and DNA could loosen the DNA helix.

Secondly, the DNA conformation in the PVA-DNA assembly was investigated by $\mathrm{CD}$ measurement. The $\mathrm{CD}$ spectrum of the pressurized DNA without PVA strongly differed from that of the nontreated DNA, indicating the conformational change of DNA during the HHP treatment. This suggests that the twisting and winding of the DNA helix induced under the HHP condition was still maintained after the HHP treatment. On the other hand, the B-DNA conformation of the PVA-DNA assembly was shown, as well as that of the nonpressurized DNA with and without PVA, indicating that the assembly of PVA and DNA could be formed without a conformational change of DNA.

Thirdly, the morphology of the PVA-DNA assembly was investigated by AFM. DNA was observed as a straight line and the DNA width was increased, suggesting that PVA was entwined with DNA. The PVA assembly was also observed as a particle on the PVA-DNA assembly. We previously reported that nanoparticles of the assembly of PVA itself were observed by scanning electron microscopy.(17) Therefore, it is considered that a part of the PVA chain interacted with DNA, and the other chain of PVA was assembled itself. For agarose gel electrophoresis of the PVA-DNA assembly, a similar pattern was observed, regardless of the PVA concentration.

From these results, it is suggested that PVA could interact with the minor or major grooves of DNA via hydrogen bonds, in which the hydroxyl group of PVA probably could be formed with the phosphate group of DNA (Fig. 6).

Finally, the nuclease resistance of the PVA-DNA assembly was investigated. The nuclease resistance of the pressurized DNA with and without PVA was shown. It is considered that the conformational change of the pressurized DNA without PVA could inhibit nuclease recognition. On the other hand, for the PVA-DNA assembly, the entwining of PVA with the DNA duplex could increase the nuclease resistance. Cationic polymers are used for gene delivery. DNA is highly condensed with a cationic polymer, resulting in a conformational change and a high nuclease resistance. Although the transfer of DNA into cells is achieved effectively, the transcription efficiency in the cell is relatively low because of the strong condensation of DNA. ${ }^{(23)}$ On the other hand, the PVA-DNA assembly may allow the recognition of a transcription factor because of the B-form of DNA. ${ }^{(20)}$

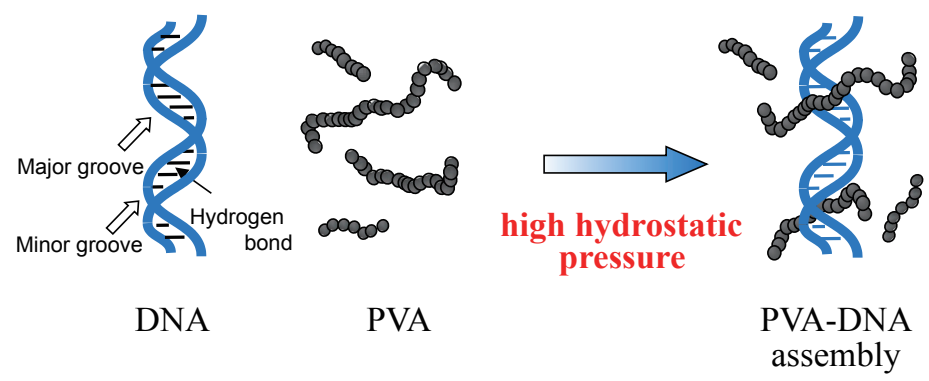

Fig. 6. (Color online) A proposed model of PVA-DNA assembly induced by HHP treatment. 


\section{Conclusions}

The PVA-DNA assembly was prepared by HHP treatment at $10000 \mathrm{~atm}$ and PVA and DNA interacted through hydrogen bonds. It was suggested that PVA was entwined with the DNA duplex, resulting in the maintenance of the B-DNA conformation. The PVADNA assembly appears to be a promising contribution to gene delivery.

\section{Acknowledgements}

This work was supported by a grant from the Ministry of Education, Culture, Sports, Science and Technology. We thank Kuraray Co. LTD. for providing poly(vinyl alcohol)s.

\section{References}

1 C. Joachim, J. K. Gimzewski and A. Aviram: Nature 408 (2000) 541.

2 Y. Cui and C. M. Lieber: Science 291 (2001) 851.

3 M. Yokoyama, M. Miyauchi, N. Yamada, T. Okano, Y Sakurai, K. Kataoka and S. Inoue: Cancer Res. 50 (1990) 1693.

4 N. Nishiyama and K. Kataoka, Pharmacol. Ther. 112 (2006) 630.

5 D. T. Bong, T. D. Clark, J. R. Granja and M. R. Ghadiri: Angew. Chem. Int. Ed. 40 (2001) 988.

6 D. Chandler: Nature 437 (2005) 640.

7 T. Tanaka, T. Tasaki and Y. Aoyama: J. Am. Chem. Soc. 124 (2002) 12453.

8 T. Zemb: Curr. Opin. Colloid Interface Sci. 8 (2003) 1.

9 J. M. Lehn: Proc. Natl. Acad. Sci. USA 99 (2002) 4763.

10 L. J. Prins, F. De Jong, P. Timmerman and D. N. Reinhoudt: Nature 408 (2000) 181.

11 J. V. Barth, J. Weckesser, N. Lin, A. Dmitriev and K. Kern: Appl. Phys. A 76 (2003) 645.

12 V. Balzani, A. Credi, F. M. Raymo and J. F. Stoddart: Angew. Chem. Int. Ed. 39 (2000) 3349.

13 V. V. Mozhaev, K. Heremans, J. Frank, P. Masson and C. Balny: Proteins Struct. Funct. Genet. 24 (1996) 81.

14 S. Kunugi, D. Yoshida and H. Kiminami: Colloid Polym. Sci. 279 (2001) 1139.

15 L. Otero and P. D. Sanz: J. Food Sci. 68 (2003) 2523.

16 M. T. Kalichevsky-Dong, S. Ablett, P. J. Lillford and D. Knorr: Int. J. Food Sci. Technol. 35 (2000) 163.

17 S. Mutsuo, K. Yamamoto, T. Furuzono, T. Kimura, T. Ono and A. Kishida: J. Polym. Sci. B 46 (2008) 743.

18 T. Kimura, A. Okuno, K. Miyazaki, T. Furuzono, Y. Ohya, T. Ouchi, S. Mutsuo, H. Yoshizawa, Y. Kitamura, T. Fujisato and A. Kishida: Mater. Sci. Eng. C 24 (2004) 797.

19 T. Kimura, S. Iwai, T. Moritan, K. Nam, S. Mutsuo, H. Yoshizawa, M. Okada, T. Furuzono, T. Fujisato and A. Kishida: J. Artif. Organs 10 (2007) 104.

20 T. Kimura, Y. Nibe, S. Funamoto, M Okada, T. Furuzono, T. Ono, H. Yoshizawa, T. Fujisato and A. Kishida: J. Drug Delivery 2011 (2011) Article ID 962743.

21 J. Q. Wu and R. B. Macgregor, Jr: Biochemistry 32 (1993) 12531.

22 R. B. Macgregor, Jr:: Biopolymers 48 (1998) 253.

23 K. Fant, E. K. Esbjorner, P. Lincoln and B. Norden: Biochemistry 47 (2008) 1732. 